\title{
Solute rejection of dextran by EVAL membranes with asymmetric and particulate morphologies
}

\author{
Liao-Ping Cheng ${ }^{a}$, Hung-Y/in Lin ${ }^{b}$, Leo-Wang Chen ${ }^{b}$ and Tai-Horng Young ${ }^{c, *}$ \\ ${ }^{a}$ Department of Chemical Engineering, Tamkang University, Taipei, Taiwan, ROC \\ ${ }^{b}$ Department of Chemical Engineering, National Taiwan University, Taipei 10764, \\ Taiwan, ROC \\ ${ }^{C}$ Center for Biomedical Engineering, College of Medicine, National Taiwan University, \\ Taipei 10016, Taiwan, ROC \\ (Received 11 March 1997; revised 9 May 1997)
}

\begin{abstract}
EVAL copolymer was precipitated from water and octanol to form, respectively, asymmetric and particulate membranes in an isothermal immersion precipitation process. Permeability of these membranes was examined with respect to dextran samples of various sizes. The results indicate that asymmetric membranes reject large dextran molecules and let through small molecules for the pressure range of $0.25-0.75 \mathrm{~kg} / \mathrm{cm}^{2}$. The particulate membranes, however, exhibit an unusual filtration behaviour; i.e. there exists a maximum rejection at intermediate dextran molecular weight. This implies that small molecules tend to be trapped inside the nano-pores within the EVAL particles whereas large molecules travel through the tortuous channels outside the particles during a filtration operation. () 1998 Elsevier Science Ltd. All rights reserved.
\end{abstract}

(Keywords: ethylene vinyl alcohol copolymer; particulate membrane; dextran)

\section{NOMENCLATURE}

$\begin{array}{ll}b & \text { stirred cell radius, } \mathrm{m} \\ C_{b} & \text { solute concentration in the feed, } \mathrm{kg} / \mathrm{m}^{3} \\ C_{f} & \text { solute concentration in the filtrate, } \mathrm{kg} / \mathrm{m}^{3} \\ C_{m} & \text { solute concentration at the membrane-solution interface, } \mathrm{kg} / \mathrm{m}^{3} \\ D_{x} & \text { solute diffusion coefficient at infinite diluticn in water, } \mathrm{m}^{2} / \mathrm{sec} \\ J_{v} & \text { filtrate flux, } \mathrm{m} / \mathrm{sec} \\ k & \text { mass transfer coefficient, } \mathrm{m} / \mathrm{sec} \\ k_{B} & \text { Boltzmann's constant, } \mathrm{J} / \mathrm{K} \\ M W & \text { molecular weight of dextran, g/mole } \\ r & \text { mean projected solute radius, } \mathrm{m} \\ R & \text { rejection coefficient for a fraction of given molecular weight } \\ & \\ \mathrm{Re} & \text { Reynolds number, } \omega^{2} / \nu \\ S_{a} & \text { actual membrane } \mathrm{sieving}^{2} \mathrm{coefficient} \\ S_{b} & \text { observed sieving coefficient } \\ \mathrm{Sc} & \text { Schmidt number, } \nu / D_{x} \\ T & \text { absolute temperature, } \mathrm{K} \\ \psi & \text { coefficient in equation }(5) \\ \mu & \text { viscosity, Pa-sec } \\ \nu & \text { kinematic viscosity, } \mathrm{m}^{2} / \mathrm{sec} \\ \omega & \text { stirring speed, rpm }\end{array}$

\section{INTRODUCTION}

The invention of asymmetric cellulose acetate membrane by Loeb and Sourirajan ${ }^{1}$ in the early 1960s is a milestone in the history of membrane science and technology. Since then, considerable progress has been made in various aspects of membrane related fields such as, development of novel membrane material, design of membrane formation process, discovery of profitable applications, etc. An asymmetrical membrane is characterised by a thin and continuous top surface layer, commonly recognised as the skin, and

* To whom correspondence should be addressed underneath which is a porous solid matrix that may be uniform or anisotropic. The skin layer is known to provide major resistance to the permeation of solute whereas the porous region functions exclusively as a mechanical support. The capability of an asymmetric membrane to reject or admit a certain solute species is, therefore, determined by the morphology, the thickness, and the pore size and density of the skin layer.

Recently, a new type of symmetric membrane, which has the so called 'particulate' morphology, has attracted special attention among our research team ${ }^{2,3}$. This membrane appears to be granular as it is observed under scanning electron microscope. It is composed of nearly equal-sized spherical particles that have interconnected to form a uniform structure. The diameter of the particles in different membranes can vary from a few hundred nanometres to several micron, depending upon the nucleation density of the particles. Unlike the asymmetric membrane, this membrane is virtually skinless. In fact, there are merely sporadic cases in the literature that describe the formation mechanism of particulate morphology during the precipitation process ${ }^{2-5}$. As far as EVAL membrane is concerned, no investigation has ever been carried out to identify the performance of the particulate structure during a filtration process.

In this work, EVAL membranes having asymmetric or particulate morphology were prepared and characterised by various techniques (e.g. SEM, mercury intrusion porosimetry, water flux and solute rejection). An interesting phenomenon was discovered: during filtration, particulate EVAL membranes tend to retain small solute molecules instead of large ones within the membrane. It is, therefore, of great importance to look into the potential applications that the particulate EVAL membranes may possess in the future. 


\section{MATERIALS AND METHODS}

\section{Material}

The membrane material studied in this work is an ethylene vinyl alcohol copolymer (EVAL) which contains ca. 56 mole \% vinyl alcohol (intrinsic; viscosity $=0.87 \mathrm{dl} / \mathrm{g}$, measured $\left.\mathrm{M}_{\eta}=56000 \mathrm{~g} / \mathrm{mole}\right)^{6}$. This polymer was kindly supplied by Kuraray Co. Ltd, Japar. Dimethyl sulfoxide (DMSO) and octanol of extra pure reagent grade (Nacalai Tesque, Kyoto, Japan) were used as received. Double distilled and de-ionised water was also used.

\section{Membrane preparation and characterisation}

Membranes were prepared using the direct immersionprecipitation method. An appropriate amount of EVAL was dissolved in DMSO to form a $25 \mathrm{wt} \%$ homogeneous solution. This solution was dispersed uniformly on a glass plate $(\mathrm{ca} .100 \mu \mathrm{m})$ by an autocoater (KCC303, RK PrintCoat Instruments, UK), then precipitated in a non-solvent bath to form a laminate. The non-solvent and residual solvent were removed from this laminate by a series of washing steps. The compositions of the non-solvent baths are summarised in Table 1. Freeze-dried samples of the membranes were examined using a scanning electron microscope (SEM) to obtain the surface and interior structures. The pore size distribution of each membrane (freeze-dried) was measured by mercury intrusion porosimetry (Micrometrics Autopore II-9220). The intrusion volume of mercury was recorded for various pressures over the range $0-50000$ psi.

\section{Filtration}

Pure water flux and solute rejection were determined using a $25 \mathrm{~mm}$ dia. Amicon Stirred Ultrafiltration Cell (Model 8010) at a stirring speed of $600 \mathrm{rpm}$. The solutes used in this work were commercial dextran fractions with average molecular weights $(\mathrm{g} / \mathrm{mole})$ of $6000(6 \mathrm{~K}), 11.5 \mathrm{~K}$, $41 \mathrm{~K}, 70 \mathrm{~K}$ and $505 \mathrm{~K}$. The $6 \mathrm{~K}$ dextran sample was purchased from Fluka and the other dextran samples were purchased from Sigma. The feed solutions were prepared by dissolving dextrans in distilled de onised water. These solutions were agitated for $24 \mathrm{~h}$ to insure complete dissolution. For all filtration experiments, the concentration of solute in the feed was taken to $\mathrm{e} 1000 \mathrm{ppm}$ and the temperature was kept at $20 \pm 3^{\circ} \mathrm{C}$. A compressed nitrogen gas tank connected to the filtration cell was used as the pressure source. Three transmembrane pressures were employed, $0.25,0.5$ and $0.75 \mathrm{~kg} / \mathrm{cm}^{2}$. In this study, low concentration feed employed with vigorous agitation under low transmembrane pressure in the filtration process is to minimize the effect of concentration polarisation. After the permeate flux reaches a stable constant value (ca. $50 \mathrm{~min}$ after operation), samples of filtrate were collected for subsequent chromatographic analysis. To check whether the membrane has any defect, a solution containing $100 \mathrm{ppm}$ blue dextran was used as the feed. The average molecular weight of this blue dextran was $2000 \mathrm{~K}$ (Sigma). All

Table 1 The non-solvents for EVAL membranes

\begin{tabular}{ll}
\hline Membrane & Coagulation bath (weight fraction) \\
\hline H1 & DMSO:Water $=0: 4$ \\
H2 & DMSO: Water $=1: 3$ \\
H3 & DMSO:Water $=2: 2$ \\
H4 & DMSO:Water $=3: 1$ \\
P1 & Octanol \\
\hline
\end{tabular}

tested membranes were found to reject the passage of blue dextran.

\section{Chromatographic analysis}

Feed and permeate samples were analysed by gel permeation chromatography (GPC) using the Bio-SECS4000 column from Phenomenex Corporation, USA. Double distilled and de-ionised water was used as the mobile phase. The effluent flow rate was maintained at $1.0 \mathrm{ml} / \mathrm{min}$ using a SPECTROFLOW 400 pump (Applied Biosystem Corporation, USA). The refractive index detector in this GPC system was Shodex RI SE-61 (Showa Denko Corporation, Japan). The molecular weight of the solute was calculated from the measured retention time, using calibration curves constructed from several runs of dextran standards (weight average molecular weight: $5 \mathrm{~K}, 12 \mathrm{~K}$ and $50 \mathrm{~K}$ obtained from Fluka).

\section{Analysis of data}

The concentration of the solute in the feed $\left(C_{b}\right)$ and the filtrate $\left(C_{f}\right)$ are related to the rejection coefficient $(R)$

$$
R=1-\frac{C_{f}}{C_{b}}
$$

The observed sieving coefficient $\left(S_{o}\right)$ of a membrane is defined as the ratio of $C_{f}$ and $C_{b}$, or

$$
S_{o}=1-R=\frac{C_{f}}{C_{b}}
$$

During filtration, solute may deposit on the membrane surface to provide an additional resistance to the transport of fluid through the membrane. In this case, a concentration boundary layer will form from the membrane surface toward the bulk. Thus, discussion of the sieving capability of a membrane must take into consideration the influence with which this extra-resistance brings. In case that agitation is employed in the feed solution such that the bulk is maintained at a constant concentration $C_{b}$, and that in the region near solution-bath interface (i.e. boundary layer), solute concentration gradually increases and reaches a maximum $\left(C_{m}\right)$ at the membrane-solution interface, then the flux of filtrate at steady state can be expressed in terms of solute concentrations $^{7,8}$

$$
\frac{C_{m}-C_{f}}{C_{b}-C_{f}}=\exp \left(\frac{J_{v}}{k}\right)
$$

where $J_{v}$ is the filtrate flux and $k$ is the mass transfer coefficient of the device. The actual membrane sieving coefficient $S_{u}$, is then defined as the ratio of the solute concentration in the filtrate $\left(C_{f}\right)$ to that at the membrane surface $\left(C_{m}\right)$. Using equation (2) and equation (3), the actual membrane sieving coefficient can be written as

$$
S_{a}=\frac{C_{f}}{C_{m}}=\frac{1}{1+\left(1 / S_{o}-1\right) \exp \left(J_{v} / k\right)}
$$

From equation (4), $S_{a}$ can be determined for a given value of $k$. For the geometry of the present filtration cell, $k$ can be estimated from an empirical correlation developed by Smith et al. ${ }^{9}$ for laminar flow in a stirred cell:

$$
\frac{k b}{D_{\infty}}=\psi(\operatorname{Re})^{0.567}(\mathrm{Sc})^{0.33}
$$

where $b$ is the radius of the stirred cell, Re is the Reynolds number and $\mathrm{Sc}$ is the Schmidt number. The coefficient $\psi$ is a function of device geometry, which is 0.23 for our 
apparatus ${ }^{10} . D_{x}$ is the diffusion coefficient of dextran at infinite dilution in water. $D_{x}$ depends upon the molecular weight of dextran $(M W)$ and can be evaluated by the correlation $^{11.12}$

$$
\log D_{x}=-4.1154-0.47752 \log (M W)
$$

equation (6) was found to be valid for the molecular weight from 21.6 to $526 \mathrm{~K}$. The unit of $D_{x}$ in equation (6) is $\mathrm{cm}^{2} / \mathrm{sec}$.
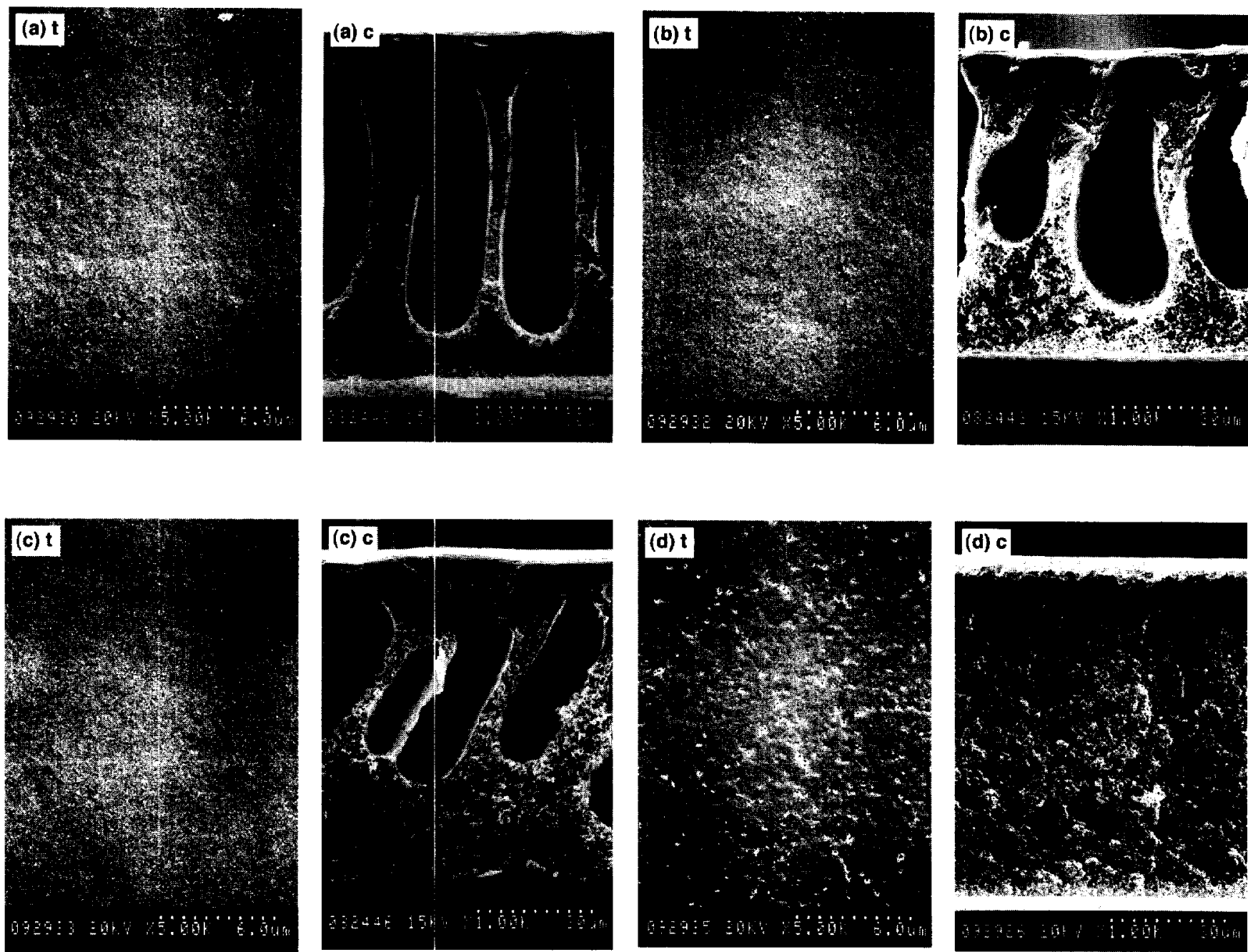
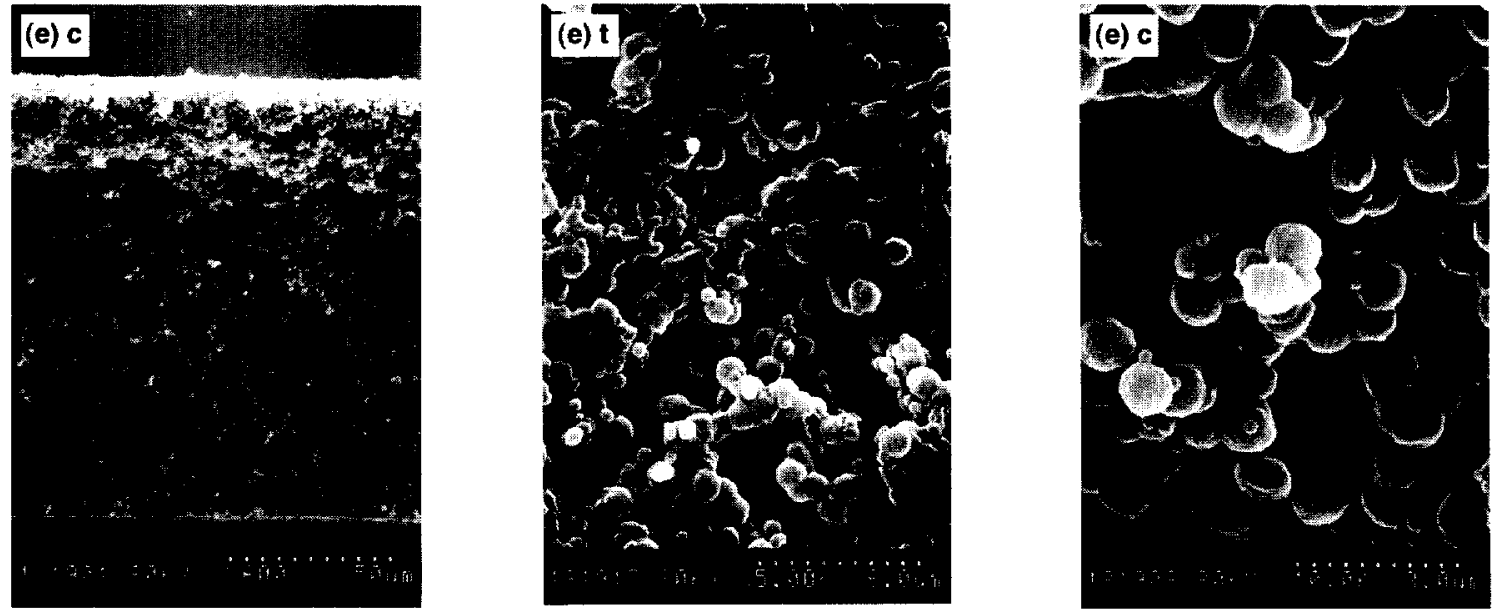

Figure 1 SEM photomicrographs of EVAL membranes: (a) $\mathrm{H1}$; (b) $\mathrm{H} 2$; (c) $\mathrm{H} 3$; (d) $\mathrm{H} 4$; (e) Pl. t: top surface view: c: cross-section view 
Solute rejection of dextran by EVAL membranes: L.-P. Cheng et al.

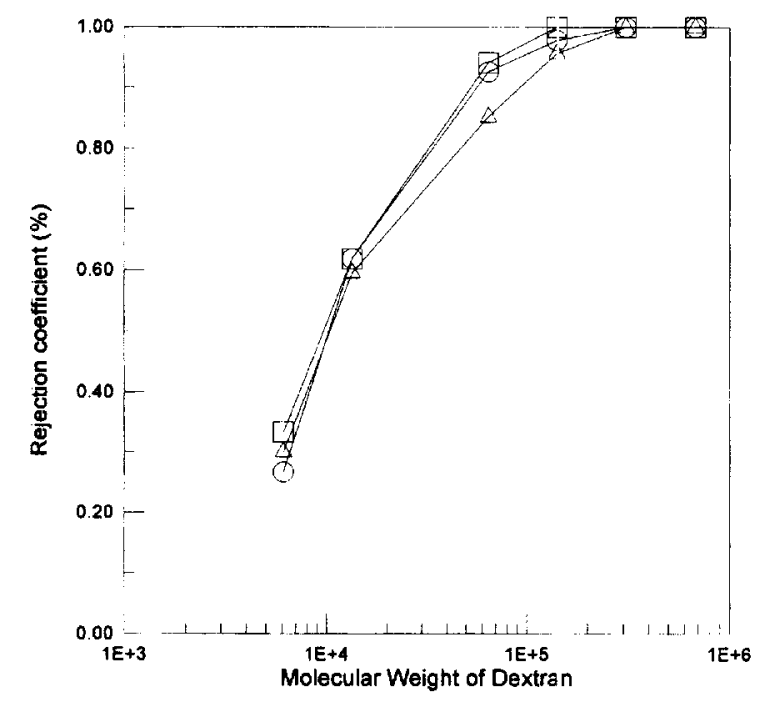

(a)

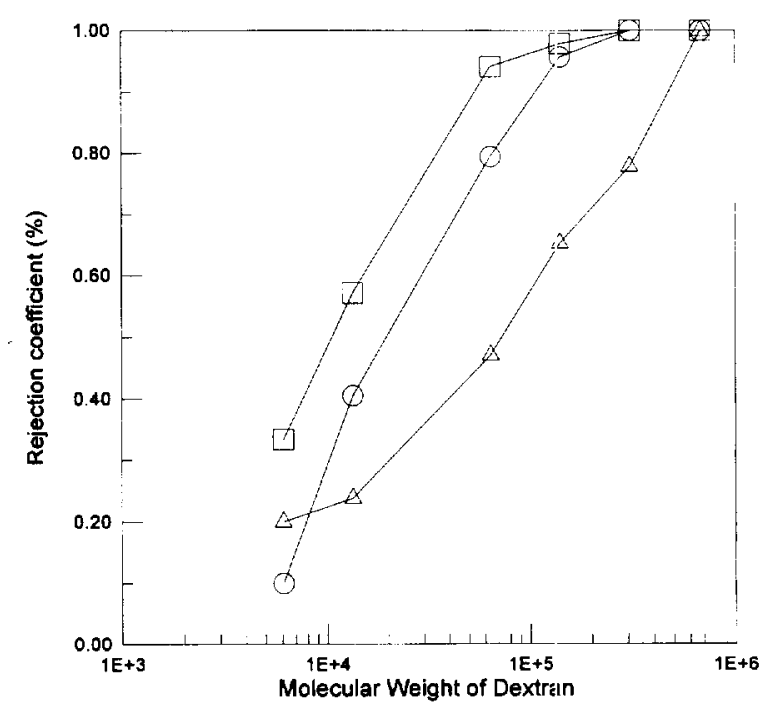

(c)

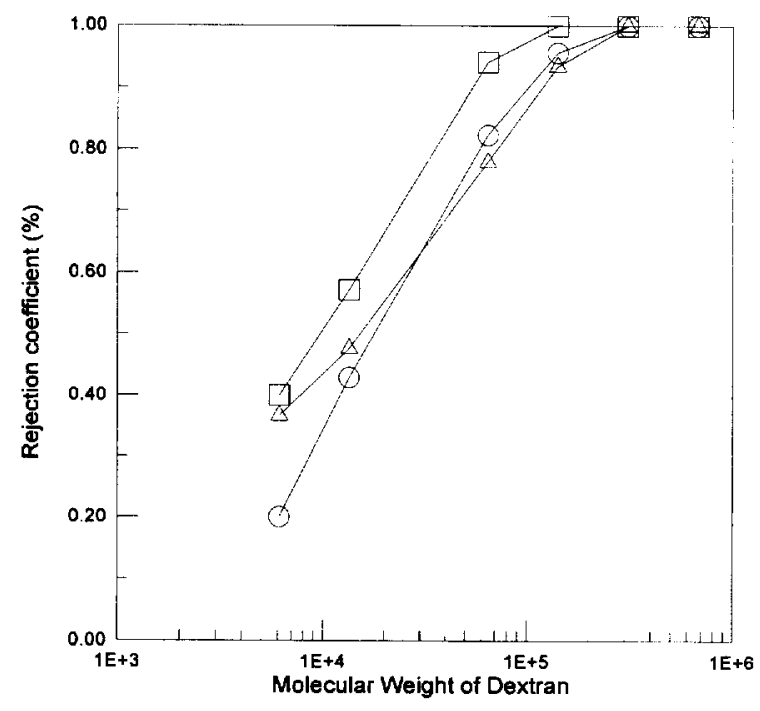

(b)

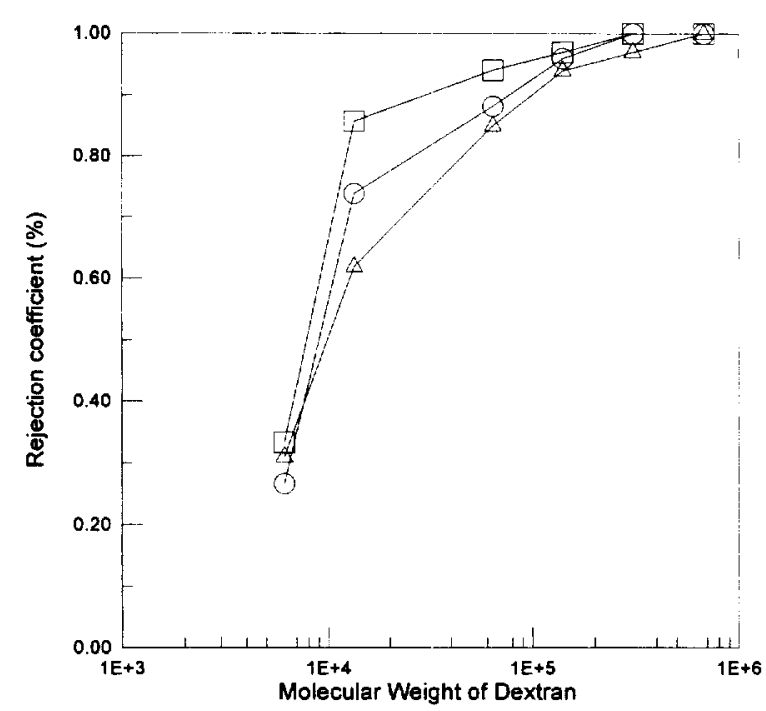

(d)

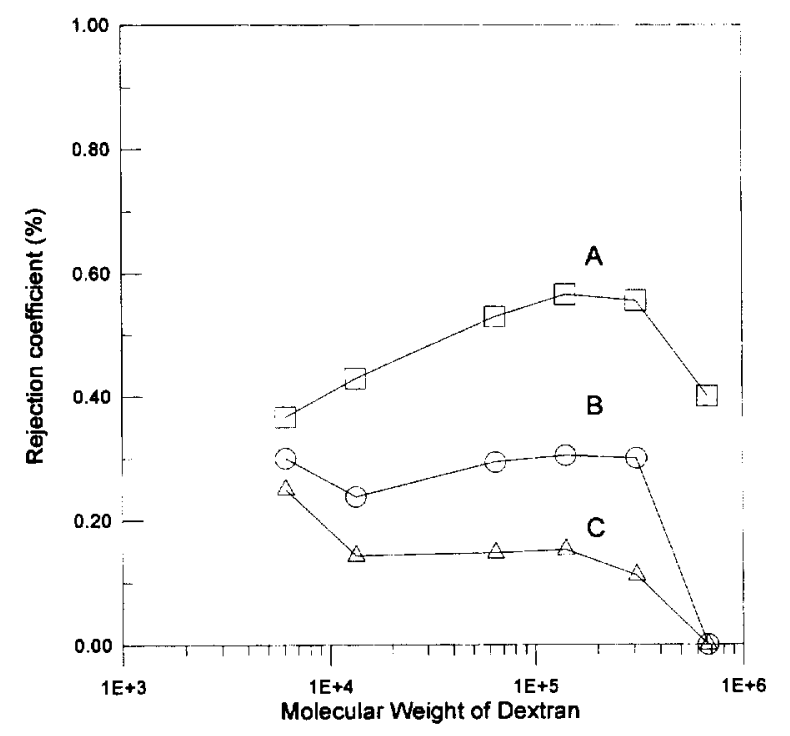

(e)

Figure 2 The rejection coefficients for filtration of the dextran mixture through different EVAL membranes as a function of the dextran molecular weight with the applied pressure of 0.25 (二), $0.5(\mathrm{O})$ and $0.75(\triangle) \mathrm{kg} / \mathrm{cm}^{2}$, respectively. (a) $\mathrm{HI}$; (b) $\mathrm{H} 2$; (c) $\mathrm{H} 3$; (d) $\mathrm{H} 4$; and (e) $\mathrm{PI}$ 
DMSO. Polymer swells to a certain degres in these baths. Hence, precipitation takes place sluggishly. Membrane P1 was prepared using octanol as the non-silvent. Because EVAL absorbs a significant amount of octanol ( $\mathrm{ca} .35 \mathrm{wt} \%$, in an absorption equilibrium experiment; at $25^{\circ} \mathrm{C}$, it is considered as a very 'soft' non-solvent for EVAL. The structures of the formed membranes were observed using SEM and the results are given in Figure la-e. Membranes $\mathrm{H} 1-\mathrm{H} 4$ show the characteristics of an as $y$ mmetric membrane being composed of a dense layer neal the top surface and a porous supporting solid matrix. As was reported previously $^{6}$, the tie-lines of the liquid-liquid phase equilibrium boundary for the EVAL-DMSO-water system had large negative slopes. As a result, the surface layer of the membrane solution will become rich in polymer soon after it contacts with the non-solvent bath ${ }^{13}$. This dense gel layer later fuses to form the continuous and virtually nonporous skin. The porous bulk of membranis $\mathrm{H} 1-\mathrm{H} 3$ have large finger-shape cavities which are often observed in membranes formed from a rapid-demixing process ${ }^{14,15}$. Membrane $\mathrm{H} 4$ was prepared by precipitation from a rather soft bath (75 wt\% DMSO). This membrane has a uniform pore distribution in its cross section. It does not contain any finger-shape macrovoids. This is consistent with the results reported by Wijmans et al. ${ }^{16}$ and Young and Chen ${ }^{17}$ that by adding solvent to the coagulation bath, the macrovoids can effectively be suppressed and that the macrovoids can be totally eliminated when the non-solvent bath contains a very high concentration of solvent. The structure of membrane $\mathrm{Pl}$, as shown in Figure le, differs dramatically from those of membranes $\mathrm{H} 1-\mathrm{H} 4$. This membrane is uniform and skinless composed of EVAL particles (ca. $(1.5 \mu \mathrm{m}$ dia.) that fuse together at the surfaces to form an interconnected solid phase. The pores are also open and connected, which make this membrane exhibit a co-continuous morphology. As will be shown later, some unusual filtration results can be derived from the internal structure within the EVAL particles.

\section{Dextran rejection}

Dextran fractions of different molecular weights were mixed and filtered through EVAL membranes for three pressures: $0.25,0.5$ and $0.75 \mathrm{~kg} / \mathrm{cm}^{2}$. The concentrations of dextran in the feed and permeate were measured and the results were substituted into equation (1) to obtain the rejection coefficients $(R)$. In Figure $2 a-d$, these rejection coefficients were plotted as a function of dextran molecular weight for the asymmetric membranes $(\mathrm{Hl}-\mathrm{H} 4)$. It can be observed that the rejection coefficients for all dextran fractions almost decrease with the transmembrane pressures. In recent publications of Balmann and Nobrega ${ }^{18}$ and Schock and Miquel ${ }^{19}$, a similar result was reported for the filtration of dextran through polysulfone and cellulose acetate membranes. At elevated pressures, dextran molecules are compressed and deformed into configurations that fit easily into the pores in the swollen skin layer. These molecules then permeate quickly through the porous region. A poor dextran rejection is therefore observed. Figure $2 a-d$ show that the rejection coefficients increase with the dextran molecular weight for all asymmetric membranes. This result agrees with the ordinary observation that small particles pass through the membrane whereas large particles are retained.

The rejection coefficient of dextran for the particulate membrane, Pl, however, shows an extraordinary trend. At the pressure of $0.25 \mathrm{~kg} / \mathrm{cm}^{2}$, as indicated in curve 'A' of Figure $2 e$, the rejection coefficient increases first and then decreases with dextran molecular weight. A maximum

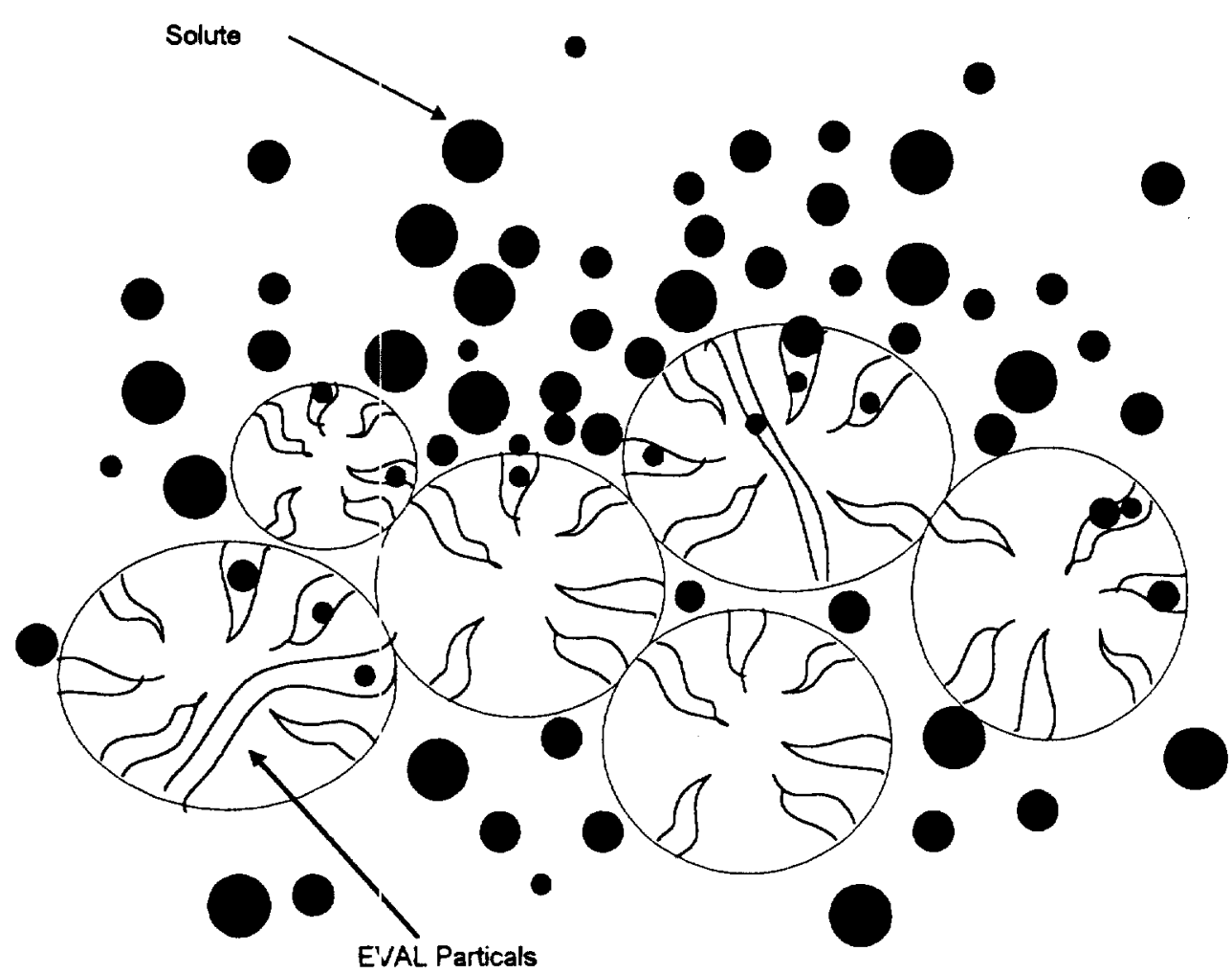

Figure 3 Schematic representation of the mechanism of solute transportation in a membrane with a particulate morphology: small molecules tend to be trapped in the pores within the particles while large mislecules can migrate out of the membrane through the tortuous channels outside the particles during a filtration operation 
occurs at dextran molecular weight equal to $140 \mathrm{~K}$ (this dextran is termed $\mathbf{M}$-dextran, hereafter). This suggests that dextran molecules of different sizes may permeate through the membrane by different mechanisms. As will be shown later, the pores of membrane P1 (measured by the mercury intrusion method) can largely be divided into two categories: the small pores (nanornetre order, termed S-pore hereafter) inside the EVAL particles and the large open pores ( $\mathrm{ca} .>0.1 \mu \mathrm{m}$, termed L-pore, hereafter) between the EVAL particles. In other words, the PI membrane is packed with internally porous particles that are fused together at the interface. Consider the case that the $S$-pores of the swollen EVAL particles have a pore diameter similar to the size of the M-dextran. Then, those dextran molecules that are larger than the M-dextran will transport through the membrane by way of the inter-connected L-pores and yield a lower rejection coefficient. On the other hand, molecules smaller than the M-dextran may diffuse into the S-pores. Once these molecules get inside the S-pores of the EVAL particles, small molecules pass through the particles faster than large molecules. Hence, the rejection coefficients increase with the molecular weight in this part of the curve. This explain!s why there exists a maximum for the rejection curve shown in Figure $2 e$. Such a separation characteristic is shown schematically in Figure 3. The large molecules are excluded from the small cavities within the EVAL particles whereas the small molecules are able to migrate into the internal voids of the particles.

At the pressures of 0.5 and 0.75 pressure $\mathrm{kg} / \mathrm{cm}^{2}$, the rejection coefficients change with the dextran molecular weight in a sigmoid manner. As shown in Figure $2 e$, the rejection curves ( $B$ and $C$ ) decrease moderately at first, then increase weakly to a maximum and finally undergo a sharp decrease and reach zero (note: these membranes reject dextrans with a molecular weight of $2000000 \mathrm{~g} / \mathrm{mole}$ ). As in the case of filtration at $0.25 \mathrm{~kg} / \mathrm{cm}^{2}$, the 2 nd (weak increase) and 3rd (sharp decrease) parts of the rejection curves suggest that there are two types of transportation paths for dextran molecules of different sizes; namely, the tortuous channels outside the EVAL prarticles and the nanovoids inside the particles. Because clextran molecules are more compact at higher pressures, the maximum rejection shifts to a higher molecular weight relative to the $0.25 \mathrm{~kg} /$ $\mathrm{cm}^{2}$ case. Curves $\mathrm{B}$ and $\mathrm{C}$ indicata a zero rejection at molecular weights approximately equal to $700 \mathrm{~K}$. Since blue dextran $(2000 \mathrm{~K})$ cannot come across membrane P1, the equivalent pore size of this membrane is between 700 and $2000 \mathrm{~K}$. The decrease of rejection coefficient at the low molecular weight part of curves B and $\mathrm{C}$ is still unexplainable at present. Although one may consider this decrease to be insignificant. Those results, however, suggest that it is related to the deformation of dextran molecule at higher pressures, at which dextran molecules become more compact so as to block the smaller pores within the particles more easily.

\section{Pure water flux versus the membrane morphology}

The pure water fluxes for those EVAL membranes shown in Table $l$ are presented as a function of transmembrane pressure in Figure 4. For the asymmetric membranes, H1$\mathrm{H} 3$, water fluxes increase, as the DMSO content in the precipitation baths is increased. Since these membranes have a similar interior structure (Figure $l a-c$ ), their water permeability is dictated by the thickness of the skin layer and how tightly this layer is packed. Therefore, Figure 4

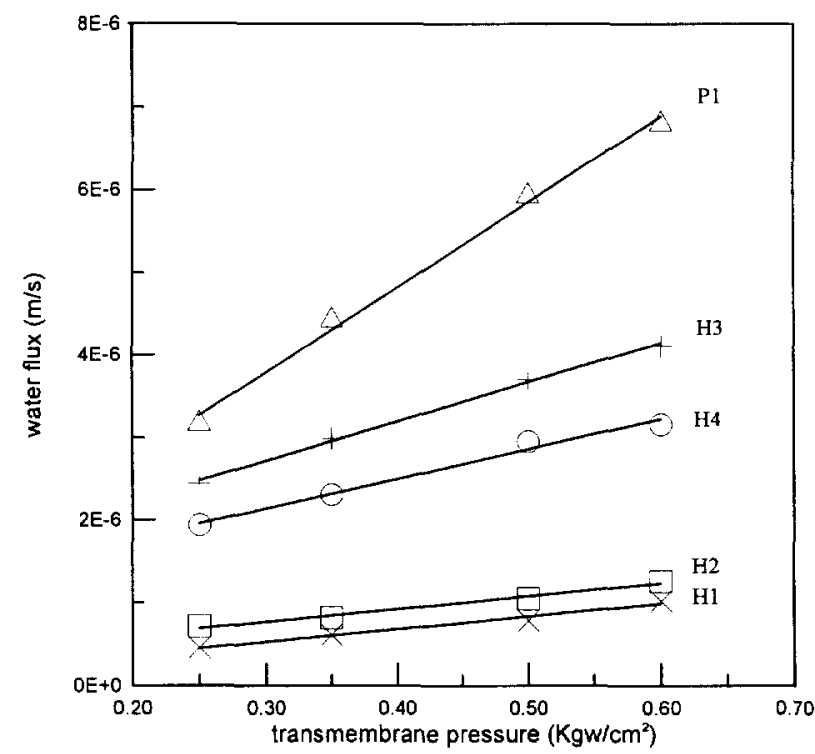

Figure 4 The pure water flux of the EVAL membranes. $\times: \mathrm{H} 1 ; \sqsupset: \mathrm{H} 2$; $+: \mathrm{H} 3 ; \mathrm{O}: \mathrm{H} 4 ; \triangle: \mathrm{PI}$

suggests that EVAL membranes prepared in a softer bath have a less dense skin layer. On the contrary, Figure 4 shows that the water permeation rate of membrane $\mathrm{H} 4$ is lower than that of membrane $\mathrm{H} 3$ even though membrane $\mathrm{H} 4$ was prepared with the softest DMSO-water bath. This seemingly paradoxical situation can be explained by the fact that membrane $\mathrm{H} 4$ does not contain finger-shape macrovoids in the membrane interior. As shown in Figure $1 d$, some of the pores in membrane $\mathrm{H} 4$ are closed. These pores are surrounded by continuous polymer walls which provide considerable resistance to water permeation. Figure 4 shows that the P1 membrane has the highest water flux. Membrane $\mathrm{P} 1$ is skinless and has a uniform particulate morphology in which all voids are interconnected. Water molecules, therefore, flow through these voids with a relatively low resistance.

\section{Pore size distribution for the membranes}

The mercury intrusion porosimetry was employed to determine the pore size distribution of each EVAL membrane. In Figure $5 a$, the measured data for membrane $\mathrm{H} 1$ is shown in terms of incremental mercury intrusion volume versus equivalent diameter of void space. This membrane has a complex pore size distribution which covers a broad range of diameters extending from a few $\mathrm{nm}$ to a few tens of $\mu \mathrm{m}$, as shown in the figure. There are three principal types of cavities that can be identified, the average sizes being $50 \mu \mathrm{m}, 80 \mathrm{~nm}$, and $10 \mathrm{~nm}$. Compared with the SEM shown in Figure $I a$, it can be deduced that the large $50 \mu \mathrm{m}$ and $80 \mathrm{~nm}$ pores correspond, respectively, to the finger-shape macrovoids and the cellular pores enclosed in the polymer matrix. The smallest pores $(10 \mathrm{~nm})$ are beyond the observable sensitivity of the SEM and are thought to be the interstitial cavities within the skin layer and act as the access for the transport of small solute particles through this skin layer. Although severe compression and deformation of polymeric membranes occurs when detecting small pores using the mercury intrusion method under enormous pressure, these data could be viewed as references if compared to the pore diameter calculated from the actual sieving coefficient, as will be mentioned later. The other asymmetric membranes $(\mathrm{H} 2-\mathrm{H} 4)$ exhibit a pore size 
distribution similar to that of membrane $\mathrm{H} 1$ (the results for membranes $\mathrm{H} 2-\mathrm{H} 4$ are not shown here).

The structure of membrane P1 is uniform and skinless, characterised by a packed bed of roughly spherical particles that extend over the entire membrane area (recall Figure 1e). The pore size distribution of this membrane is shown in Figure $5 b$. As in the case of the asymmetric membranes, Figure $5 b$ indicates three major peaks, the equivalent diameters of which are $50 \mu \mathrm{m}, 0.3 \mu \mathrm{m}$ and $10 \mathrm{~nm}$, respectively. The two larger cavities which represent the pores between EVAL particles are associated with the packing patterns of the particles. As shown in Figure le, all large pores (ca. $2 \mu \mathrm{m}$ dia.) are, in fact, interconnected to form a continuous void space. This then leads to the deceptively large $50 \mu \mathrm{m}$ result obtained from the mercury intrusion measurement. Since EVAL particles are $0.5 \mu \mathrm{m}$ dia., the $0.3 \mu \mathrm{m}$ dia. cavity depicts reasonably the space between adjacent particles. In addition, since this membrane does not have a dense skin layer, the nano-voids are most

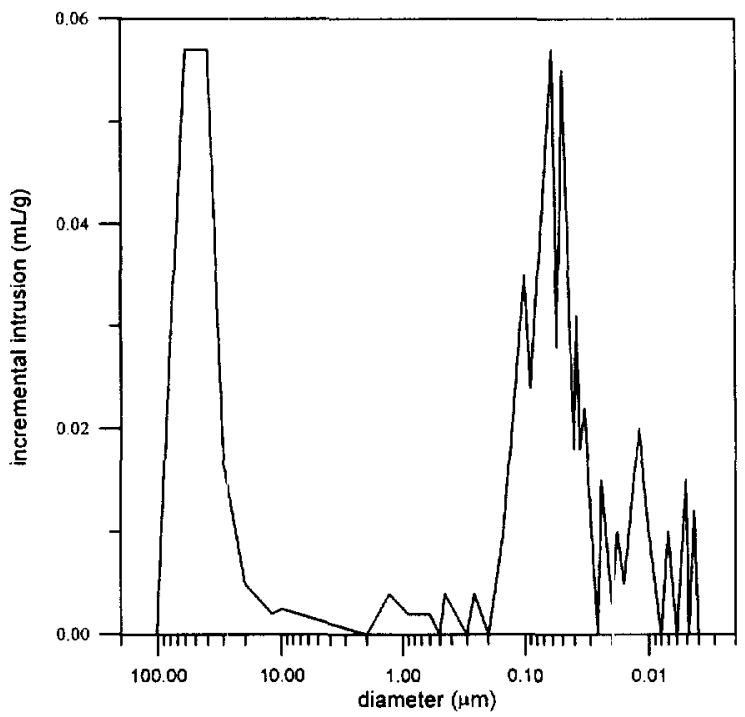

(a)

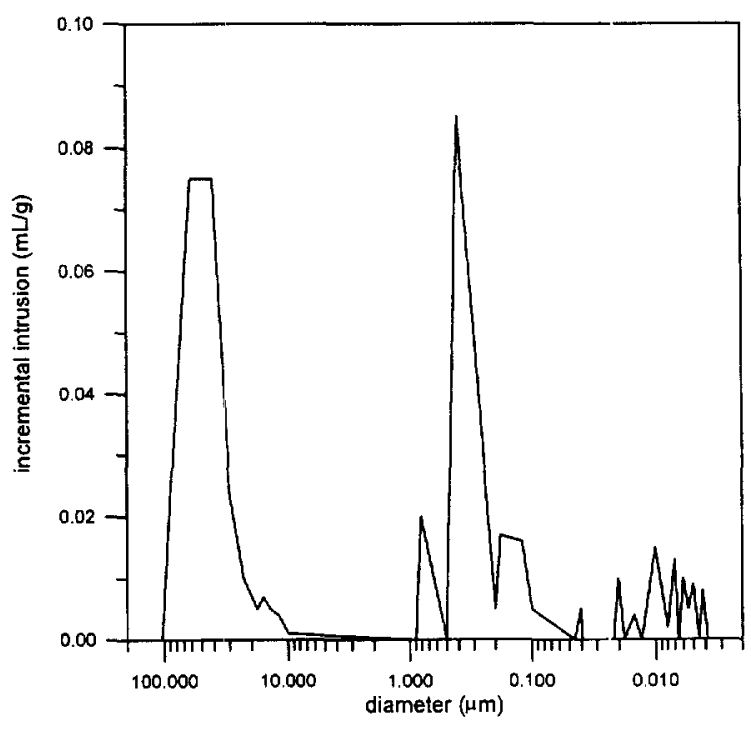

(b)

Figure 5 The pore size distribution of EVAL membranes: (a) H1; (b) P1 likely those pores inside the EVAL particles, as described previously.

\section{Pore diameter from actual sieving coefficient}

In a filtration process, retained solutes tend to accumulate on the membrane surface to form a concentration boundary layer. Such an effect, commonly known as 'concentration polarisation', can cause the back-diffusion of solute toward the bulk and thus, result in inefficient filtration. In this work, low concentration feed were employed with vigorous agitation in the filtration process, which was believed to minimize this concentration polarisation effect. Meanwhile, the actual sieving coefficients [defined in equation (4)] were determined and compared with the observed sieving coefficient [defined in equation (2)] to validate the obtained results. During calculation, the diffusion coefficient, $D_{x}$, for each of the dextran was evaluated using equation (6). This result was then substituted into equation (5) to yield the mass transfer coefficient, $k$, with which the actual sieving coefficient, $S_{a}$, can be determined. In Table 2, some typical calculated actual sieving coefficients for the $0.25 \mathrm{~kg} / \mathrm{cm}^{2}$ case are shown together with the observed sieving coefficients. It can be seen that these two sets of data are virtually the same, which implies that concentration polarisation is insignificant and can be ignored in this work.

An attempt is made to evaluate, from the actual sieving coefficient, the diameter of the pores within the skin of the asymmetric membranes $(\mathrm{H} 1-\mathrm{H} 4)$ and that within the EVAL particles of the particulate membrane $(\mathrm{P} 1)$. These pores are on the order of $\mathrm{nm}$ and can not be observed directly by regular optical means. In order to do this evaluation, the following assumptions were made: (1) The size of the largest pore in the skin of membranes $\mathrm{HI}-\mathrm{H} 4$ can be approximated by the diameter of dextran molecules at sieving coefficient approaching zero while that within the EVAL particles of membrane $\mathrm{P} 1$ is at the maximum of the dextran rejection coefficient; (2) Since there is no appropriate correlation describing dextran deformation at fluxes on the order of $10^{-6} \mathrm{~m} / \mathrm{sec}^{12}$, the mean projected solute radius $(r)$ is taken to be the Stokes radius, i.e. $r=k_{\mathrm{B}} T / 6 \pi \mu D_{\infty}$ where $k_{\mathrm{B}}$ is the Boltzmann constant, $T$ is absolute temperature, and $\mu$ is the viscosity of water at temperature $T$. For asymmetric membranes $\mathrm{Hl}$ and $\mathrm{H} 2$, the dextran molecular weight corresponding to $S_{a}=0(R=1)$ is $140 \mathrm{~K}$, as obtained from the measured rejection data given in Figure 2(a) and (b). Likewise, the dextran molecular weight at $S_{a}=0$ for membranes $\mathrm{H} 3$ and $\mathrm{H} 4$ is $300 \mathrm{~K}$, obtained from Figure 2(c) and (d). These values were substituted into equation (6) to obtain the diffusion coefficients, which were then used with the Stokes Equation to give the projected solute radius, $r$. The calculated $r$ values are 8 and $11 \mathrm{~nm}$, respectively, for each case. Hence, the diameter of the largest pore in the skin layers of membranes $\mathrm{H} 1$ and $\mathrm{H} 2$ is approximately $16 \mathrm{~nm}$ while that of membranes $\mathrm{H} 3$ and $\mathrm{H} 4$ is $22 \mathrm{~nm}$. For membrane $\mathrm{Pl}$, the largest pore within the EVAL particles is found to be $16 \mathrm{~nm}$ since the maximum of the rejection coefficient occurring at dextran molecular weight is equal to $140 \mathrm{~K}$. These results appear to

Table 2 Comparison of the observed and actual sieving coefficients for dextran $(13 \mathrm{~K})$ at $0.25 \mathrm{~kg} / \mathrm{cm}^{2}$

\begin{tabular}{|c|c|c|c|c|c|}
\hline Membrane & $\mathrm{H} 1$ & $\mathrm{H} 2$ & $\mathrm{H} 3$ & $\mathrm{H} 4$ & P1 \\
\hline$S_{0}$ & 0.381 & 0.429 & 0.429 & 0.143 & 0.571 \\
\hline$S_{a}$ & 0.382 & 0.428 & 0.428 & 0.142 & 0.571 \\
\hline
\end{tabular}


Solute rejection of dextran by EVAL membranes: L.P. Cheng et al.

agree with the nanometre order cavities obtained from the mercury intrusion measurements, shown in Figure $5 a$ and $b$.

\section{CONCLUSIONS}

In this work, EVAL copolymer were precipitated from nonsolvent in the immersion-precipitat on process to form membranes exhibiting either asymrnetric or particulate morphology. The asymmetric membrane is composed of a skin and a porous supporting layer. The particulate membrane is uniform and skinless composed of a packed bed of fused spherical particles that are of similar size ( $\mathrm{ca}$. $0.5 \mu \mathrm{m}$ dia.). The following points are deduced from careful examination of the experimental results.

(1) As in other membrane forming systems, EVAL membranes precipitated from a 'soft' coagulation bath will feature a less compact skin and smaller finger-shape voids than those precipitated from the 'harsh' water bath.

(2) There exist pores inside the skin of the asymmetric EVAL membranes, whose average diameter is on the order of nanometre. These pores dictate the selectivity of the membrane toward the solute (dextran) molecules.

(3) For the pressure range $\left(0.25-0.75 \mathrm{~kg} / \mathrm{m}^{2}\right)$ used in this work, all asymmetric membranes have a higher solute rejection and a lower water flux than the particulate membrane.

(4) The rejection coefficients for the particulate membrane indicate a maximum at intermediate dextran molecular weights. This suggests that small dextran molecules tend to be trapped in the nano-voids within the EVAL particles while large dextran molecules can migrate out of the membrane through the tort sous channels outside the particles during a filtration operation.

Besides dextran, solute rejection of polyethylene glycol and polyacrylic acid sodium salt by farticulate membranes is being studied in our laboratory and has the similar phenomenon. Therefore, the particulate structure reveals a new filtration mechanism and more efforts to understand the performance of this membrane (for example, the effect of particle size, the effect of packing degree of particles, ...) might be necessary in further study.

\section{ACKNOWLEDGEMENTS}

The authors thank the National Science Council of the Republic of China for their financial support, project numbers: NSC 85-2216-E-002-002 and NSC 85-2216-E032-001.

\section{REFERENCES}

1. Loeb, S. and Sourirajan, S., Adv. Chem. Ser., 1963, 38, 117.

2. Cheng, L. P., Dwan, A. W. and Gryte, C. C., J. Polym. Sci., Polym. Phys., 1995, 33, 211

3. Young, T. H., Chen, L. W. and Cheng, L. P., Polymer, 1996, 37, 1305

4. Cheng, L. P., Dwan, A. W. and Gryte, C. C., J. Polym. Sci., Polym Phys., 1995, 33, 223.

5. Cheng, L. P., Dwan, A. W. and Gryte, C. C., J. Polym. Sci., Polym. Phvs., 1994, 32, 1183.

6. Young, T. H., Lai, J. Y., You, W. M. and Cheng, L. P., I. Membrane Sci., 1997, 128, 55.

7. Blatt, W. F., Dravid, A., Michaels, A. S. and Nelsen, L., Membrane Science and Technology, ed. J. E. Flinn. Plenum Press, New York, 1970, pp. 47-97.

8. Mulder, M., Basic Principles of Membrane Technology. Kluwer, Dordrecht, The Netherlands, 1991.

9. Smith. K. A., Colton, C. K., Merrill, E. W. and Evans, L. B., Chem Eng. Progr. Symp. Ser, 1968, 64, 45.

10. Opong, W. S. and Zydney, A. L., AIChE J., 1991, 37, 1497.

11. Granath, K. A., J. Colloid Sci., 1958, 13, 308.

12. Mochizuki, S. and Zydeny, A. L., J. Membrane Sci, 1992, 68, 21.

13. Cheng, L. P., Soh, Y. S., Dwan, A. W. and Gryte, C. C., I. Polym. Sci., Polym. Phys., 1994, 32, 1413.

14. Reuvers, A. J. and Smolders, C. A., J. Membrane Sci., 1987, 34. 67.

15. Young, T. H. and Chen, L. W., Desalination, 1995, 103, 233.

16. Wijmans, J. G., Baaij, J. P. B. and Smolders, C. A., J. Membrane Sci., 1983, 14, 263.

17. Young, T. H. and Chen, L. W., J. Membrane Sci., 1991, 57, 69

18. de Balmann, H. and Nobrega, R., J. Membrane Sci, 1989, 40, 311

19. Schock, G. and Miquel, A., J. Membrane Sci., 1989, 41, 55. 